\title{
Novel therapeutic strategies for spinal muscular atrophy
}

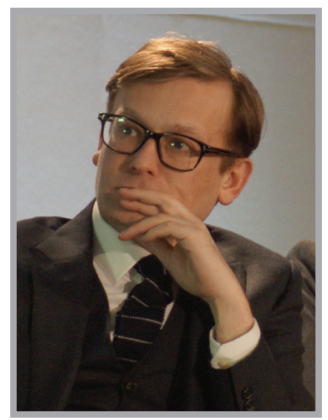

Omar Khwaja* speaks to Ellen Clarke, Commissioning Editor: Omar is a pediatric neurologist with specialist skills in critical care, fetal-neonatal neurology and neurogenetics. His research background includes human genetics and neurosciences as well as developmental neurobiology. He also has significant experience in clinical-translational research specifically related to diseases of the developing brain. Prior to Omar's current role in industry, he designed and implemented novel neurotherapeutic strategies for Rett syndrome and associated autism spectrum disorders, in addition to his clinical and academic responsibilities.

\begin{abstract}
Q How did you develop an interest in the field of spinal muscular atrophy research?

I am a pediatric neurologist and specifically specialized in neurological and genetic disorders that affect infants. Spinal muscular atrophy (SMA) most frequently affects children under the age of 1 who also have the most severe form of the disease. The disease is usually fatal when diagnosed in infancy. The genetic cause of SMA was identified in the early 1990s, but advances in understanding the biology of the disease in the last 5-10 years has led to a number of potential approaches to treat SMA.
\end{abstract}

\section{Q Currently what are the biggest challenges in the field?}

The biggest challenge in the area of SMA is that there is currently no cure or approved treatments for the neuromuscular disease. Given that SMA affects 1 in approximately 10,000 live births and is associated with a high rate of childhood mortality in its most severe forms, the disease is associated with huge unmet need for which we are limited to trying to manage disease symptom and prevent complications.
However, the area of SMA has seen huge research advances in recent years since the discovery of the genetic cause of the disease and this has led to the development of numerous approaches which may yield effective therapies in the future.

Q Can you explain a little about the role of genetics in disease onset?

SMA is a genetic disease caused by mutation or deletion of the SMN1 (survival of motor neuron) gene and is the leading genetic cause of death in infants and toddlers. It is characterized by insufficient levels of SMN protein, leading to progressive loss of motor neurons, muscle weakness and atrophy.

Patients with SMA depend on the SMN2 gene as the source of SMN protein as they have no SMN1 gene. However, only $10-30 \%$ of the mRNA produced by the normal SMN2 gene is full-length and encodes $S M N$ protein. This means patients with SMA experience a profound lack of this protein.

Q What was the rationale behind the Roche RG7800 Phase la trial? Can you

*Head of Rare Diseases, Roche Pharma Research \& Early Development, Roche Innovation Center Basel, 4070 Basel, Switzerland; omar.khwaja@roche.com 
explain a little about the preceding studies? In 2014, we published preclinical data in Science noting the identification of compounds that selectively modify SMN2 splicing which were suitable for oral administration. As part of this study, we sought to determine if increasing SMN protein levels in mouse models of SMA could improve the deficits observed in these animals. We demonstrated that splicing modifiers increase SMN protein levels in multiple tissues, providing a benefit in SMA mouse models, including an increase in life span, normalized body weight gain, improvement of motor behavior and correction of neuromuscular deficits lasting into adulthood.

The field of RNA splicing has been questioning whether or not it is possible in principle to selectively modify alternative splicing of a particular pre-mRNA with a small molecule. To the best of our knowledge, this study was the first example demonstrating that this is possible.

Following the completion of this trial, we initiated a Phase Ia single-ascending-dose, placebo-controlled, double-blind study investigating the safety, tolerability, pharmacokinetics and pharmacodynamics of RG7800 in 48 healthy subjects. The primary objective of the trial was to characterize the safety and tolerability of RG7800 in healthy subjects, alongside evaluating the pharmacokinetic and pharmacodynamic properties of the investigational compound.

\section{Q What impact will the results of the study} have on the field?

The results from this Phase Ia trial showed that RG7800 was well tolerated across the single-dose range of 0.5 to $90 \mathrm{mg}$. Proof-of-mechanism of the compound was demonstrated by the increased production of full-length SMN2 mRNA in healthy subjects in a dose-dependent manner.

This trial is an important step in SMA research as it is the first time that a small molecule $S M N 2$ splicing modifier has been shown to increase production of full-length $S M N 2 \mathrm{mRNA}$ in humans.

The safety, tolerability, pharmacokinetics and pharmacodynamics of RG7800 in adult and pediatric patients with SMA are currently being investigated in a 12-week trial (MOONFISH). This trial is expected to complete in 2016.

Q What did the study reveal about the mechanisms of RG7800?

This trial showed that up to a $100 \%$ increase in full-length $S M N 2 \mathrm{mRNA}$ was observed upon treatment with RG7800 with the compound having no effect on SMN1 mRNA in healthy subjects. This was an important finding given RG7800 is not designed to impact the SMN1 gene.

The translatability of these results in patients with SMA is being explored in the MOONFISH trial, which is investigating the safety, tolerability, pharmacokinetics and pharmacodynamics of RG7800 in adult and pediatric patients with SMA.

Q Are there any other avenues that Roche are currently exploring for the treatment of spinal muscular atrophy?

In 2015, we acquired a compound called olesoxime (RG6083), an investigational medicine being tested for its potential ability to preserve healthy motor nerve cells and which, if approved, could be the first medicine specifically developed for people with SMA.

Olesoxime is a cholesterol-like compound that binds to proteins in cells and is designed to reduce the release of cell death factors and promote the survival of motor cells. The compound is believed to work by preserving the function of mitochondria, the powerhouse of cells. Results from a 2-year Phase II/III clinical trial involving people with type II and nonambulatory type III SMA suggests that olesoxime stabilized neuromuscular function over 2 years, compared with placebo.

Q How do you see the field developing in the next 5 years?

I think this is a very exciting time to be in SMA research. Discoveries underlying the genetics and biology of SMA are creating new opportunities for drug development. At the same time, they are creating new questions about the condition such as; what factors modify the presentation of the disease? Is there an optimum window for treatment? How much clinical improvement can be expected? Where exactly in the body is SMN protein needed to give improvement? Within the next 5 years I think we will have first answers to some of these questions. Hopefully we will also have one or more effective treatments that stabilize or improve the motor and respiratory symptoms of the year.

\section{Disclaimer}

The opinions expressed in this interview are those of the 
interviewee and do not necessarily reflect the views of Future Medicine Ltd.

Financial \& competing interests disclosure

The author is employed by Roche, the sponsor of RG7800 clinical development. The author has no other relevant affiliations or financial involvement with any organization or entity with a financial interest in or financial conflict with the subject matter or materials discussed in the manuscript apart from those disclosed.

No writing assistance was utilized in the production of this manuscript. 